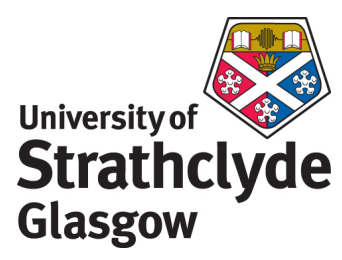

Strachan, S.M. and McArthur, S.D.J. and Stephen, B. and McDonald, J.R. and Campbell, A. (2007) Providing decision support for the condition-based maintenance of circuit breakers through data mining of trip coil current signatures. IEEE Transactions on Power Delivery, 22 (1). pp. 178-186. ISSN 0885-8977

http://strathprints.strath.ac.uk/27651/

This is an author produced version of a paper published in IEEE Transactions on Power Delivery, 22 (1). pp. 178-186. ISSN 0885-8977. This version has been peer-reviewed but does not include the final publisher proof corrections, published layout or pagination.

Strathprints is designed to allow users to access the research output of the University of Strathclyde. Copyright $(C$ and Moral Rights for the papers on this site are retained by the individual authors and/or other copyright owners. You may not engage in further distribution of the material for any profitmaking activities or any commercial gain. You may freely distribute both the url (http://strathprints.strath.ac.uk) and the content of this paper for research or study, educational, or not-for-profit purposes without prior permission or charge. You may freely distribute the url (http://strathprints.strath.ac.uk) of the Strathprints website.

Any correspondence concerning this service should be sent to The Strathprints Administrator: eprints@cis.strath.ac.uk 


\title{
Providing Decision Support for the Condition- Based Maintenance of Circuit Breakers through Data Mining of Trip Coil Current Signatures
}

\author{
Scott M. Strachan, Stephen D.J. McArthur, Member, IEEE, Bruce Stephen \\ James R. McDonald, Senior Member, IEEE, Angus Campbell
}

\begin{abstract}
The focus of this paper centers on the condition assessment of $11 \mathrm{kV}-33 \mathrm{kV}$ distribution circuit breakers from the analysis of their trip coil current signatures captured using an innovative condition monitoring technology developed by others. Using available expert knowledge in conjunction with a structured process of data mining, thresholds associated with features representing each stage of a circuit breaker's operation may be defined and used to characterize varying states of circuit breaker condition. Knowledge and understanding of satisfactory and unsatisfactory breaker condition can be gained and made explicit from the analysis of captured trip signature data and subsequently used to form the basis of condition assessment and diagnostic rules implemented in a decision support system, used to inform condition-based decisions affecting circuit breaker maintenance. This paper proposes a data mining method for the analysis of condition monitoring data, and demonstrates this method in its discovery of useful knowledge from trip coil data captured from a population of SP Power System's in-service circuit breakers. This knowledge then forms the basis of a decision support system for the condition assessment of these circuit breakers during routine trip testing.
\end{abstract}

Index Terms-Circuit breakers, monitoring, trip coil current signature, data mining, condition-based maintenance, decision support system

\section{INTRODUCTION}

$\mathrm{H}$ istorically, UK power systems were considered 'gold plated', as electrical utilities considered the health of the system and its assets to be the overriding priority as they sought to provide the public with a reliable and secure service at an affordable price. Deregulation has seen a noticeable shift in the priorities of utilities within an increasingly competitive and regulated marketplace.

While the health of the system remains a critical aspect of the business, the repercussions of privatization has seen

Manuscript received May 12, 2006

This work was supported by SP Power Systems, UK.

Dr. S. Strachan is a Research Fellow at Strathclyde University, Glasgow, UK (+44 141548 2609, scott.strachan@strath.ac.uk).

Dr. S. McArthur is a Senior Lecturer at Strathclyde University, Glasgow, U.K. Dr. B. Stephen is a Research Fellow at Strathclyde University, Glasgow, U.K. Prof. J. McDonald is the Director of the Institute for Energy and Environment at Strathclyde University, Glasgow, UK.

Mr. Angus Campbell is an asset manager at SP Power Systems, Bellshill, UK. utilities forced to become more proactive in risk mitigation and more fiscally astute than ever before.

A balance is therefore required between the conflicting objectives of satisfying customer, regulatory and shareholder demands. Asset management and suitable maintenance strategies, focusing on achieving the best possible financial and operational return on a utility's asset base, are subsequently becoming areas of increasing interest within the electricity supply industry. This has led to a proliferation of condition monitoring technologies within the industry as utilities reassess the processes, technologies and tools they employ to manage the whole life impact of costs, performance and risks associated with their ageing asset base [1].

Condition monitoring technology enables the periodic or continuous acquisition and measurement of data characterizing the 'vital signs' of operational plant. Typically, domain experts are then required to interpret this data to assess plant health and/or predict incipient failures, and where possible diagnose the root cause of any plant failure or observed deterioration in its condition. In addition to monitoring the welfare of individual plant items, the wider role of condition monitoring may be considered one of informing and supporting utility asset management and maintenance strategies.

The emergence of these strategies as key business objectives has resulted in significant investment by utilities in novel condition monitoring sensor, data acquisition and communications technologies, designed to detect and capture key plant parameters in larger volumes, at a faster rate, using less bandwidth [2]. While this undoubtedly presents plant experts with a rich vein of data, experts are charged with the onerous task of effectively managing and interpreting large volumes of data in order to gain a clear insight into the health of the plant. Overwhelming plant experts with rafts of unfamiliar plant data for the extraction of meaningful diagnostic information is clearly something to be avoided. The implications for utilities of mismanaging condition monitoring data is that the diagnostic potential of the data remains unfulfilled and the strategic decisions affecting plant operation, maintenance and replacement subsequently become less well informed.

In addition, as utilities continue to downsize, outsource, lose and retire staff, they run the risk of losing the specialist 
knowledge and experience required for plant condition assessment and defect diagnosis. All this precipitates the need for new methods of analyzing and interpreting condition monitoring data to alleviate the burden placed on what is widely regarded as a diminishing population of plant experts. In particular, where new plant parameters are made available from novel condition monitoring technologies, a means of actively driving diagnostic knowledge out of this data is required.

In conjunction with plant experts, the causal relationships between data observations and known plant behavior may be determined, modeled or encoded in rules, enabling meaningful diagnostic information to be interpreted directly from condition monitoring data captured in future. Knowledge implicit in condition monitoring data may be extracted and made explicit using a structured method of data mining tailored to meet the specific goals of classification and prediction common to condition monitoring. This 'discovered knowledge' may then be embedded within a decision support system assisting plant experts (or indeed non-experts) in future plant condition assessment and defect diagnosis.

This paper proposes a structured method of analyzing circuit breaker trip coil current data (or trip signatures) obtained from SP Power System's population of in-service distribution circuit breakers.

The outcome of this analysis is the discovery of useful domain knowledge relating to the condition assessment and defect diagnosis of $11 \mathrm{kV}-33 \mathrm{kV}$ distribution circuit breakers. This knowledge is then deployed through the development of a decision support system, assisting maintenance staff in the condition assessment of these circuit breakers during routine trip testing. The system discussed in this paper was specifically designed to provide a simplistic Red/Amber/Green output because the organization's population of field technicians had no prior knowledge or experience of how to interpret the breaker's condition from the captured trip signature. The system subsequently supports field staff in making decisions affecting the circuit breaker maintenance based on the automated assessment of the breaker condition.

The structured method of data mining and knowledge discovery applied to the condition monitoring data described in this paper operates on actual field data captured from inservice circuit breaker plant. This allows maintenance and asset management decisions to be targeted at both a strategic level (i.e. prioritization of substations and circuit breakers requiring maintenance) and an operational level (i.e. identifying specific maintenance actions required for a particular circuit breaker). The outcomes of the data mining and knowledge discovery method demonstrated in this paper may subsequently be used to inform policy making affecting asset management and condition-based maintenance practices at the strategic level. In addition, the development of a suitable decision support system for plant condition assessment, diagnosis and maintenance effectively supports the implementation of these policies and strategic decisions in the field, at an operational level.

\section{CIRCUIT BREAKER CONDITION MONITORING APPLICATION}

Circuit breakers are expected to protect circuits and plant by interrupting short circuit current within a time in the region of $100 \mathrm{~ms}-160 \mathrm{~ms}$ (depending upon the system frequency). Condition monitoring of these plant items typically involves the removal of the circuit breaker from service for off-line testing involving power factor, insulation and timing tests to assess the insulation system, contact resistance and main contact operating times of the breaker [3]. These tests concentrate mainly on the erosive effects of arcing on the breaker's main contacts and insulation during contact separation. However deterioration in a circuit breaker's condition also results from its inertia [4], where the effects of 'stiction' arising due to long periods of inactivity can result in the failed or slow operation of the breaker when it is most needed, i.e. in response to a circuit fault.

The generally quiescent nature of the circuit breaker device is such that it can typically lay dormant for long periods of time until required to interrupt load or fault current. Breakers left idle between trip operations and suffering the effects of stiction, can have their operating mechanisms exercised to effectively 'warm up' the breaker, increasing its operating speed thereafter. However, the underlying problem $/ \mathrm{s}$ responsible for this slow operation, often associated with deterioration of moving mechanical parts resulting from frictional forces, coagulation of lubricant, etc. remain disguised and subsequently neglected. This makes the circuit breaker one of the most important, yet most unpredictable plant items on the power network.

Routine trip testing, as part of a time-based maintenance strategy, has been employed historically with relative success in reducing plant failure rates, but at a relatively high cost. A more cost-effective approach to the maintenance of these circuit breakers may be achieved through condition-based decision-making during routine maintenance. The costs associated with servicing (oil replacement, lubrication, etc.) and maintaining healthy circuit breakers unnecessarily may be avoided through a more focused condition-based maintenance (CBM) strategy intended to "do only what is required, when required".

Measurement of a breaker's trip signature provides a useful insight into the operation of the circuit breaker and its condition. In addition to its primary function of controlling the breaker trip operation, the trip coil performs a secondary role as a non-invasive condition monitoring sensor, capturing the trip coil current (signature) from the 'all-important' first trip for subsequent breaker condition assessment [5]. Prior to the use of a portable trip signature recorder it was necessary to remove the breaker from service before assessing its condition and readiness to trip. Now using the handheld device to capture the trip time (i.e. the time from trip initiation until current ceases to flow in the load side of the circuit breaker) SP Power Systems based their decision regarding the 
maintenance of the breaker on the simple heuristic - "IF trip time $>100 \mathrm{~ms}$ THEN maintain breaker", irrespective of model type and manufacturer. This approach ignored the nuances associated with different circuit breaker designs and the conditions affecting the timings associated with the various stages of the breaker operation during the all-important initial trip required to remove it from service.

\section{CIRCUIT BREAKER OPERATION AND TRIP COIL CURRENT SIGNATURE}

The control or trip unit of the circuit breaker transforms the trip signal into the physical operation of the breaker mechanism. The trip coil consists of a conducting coil wrapped around a movable iron plunger forming an electromagnetic actuator. The iron plunger eventually dislodges the trip latch via a non-magnetic tappet (sitting on top of the plunger) and a trip bar, and ultimately activates the main latch, unlocking the operating shaft and discharging the opening spring causing the main contacts of the breaker to open (Figure 1) [6].

On the initiation of a trip command the trip coil becomes energized by the substation battery supply. As the trip coil is energized the sequence of events illustrated in Figure 1 are initiated, leading to the opening of the circuit breaker main and auxiliary contacts.

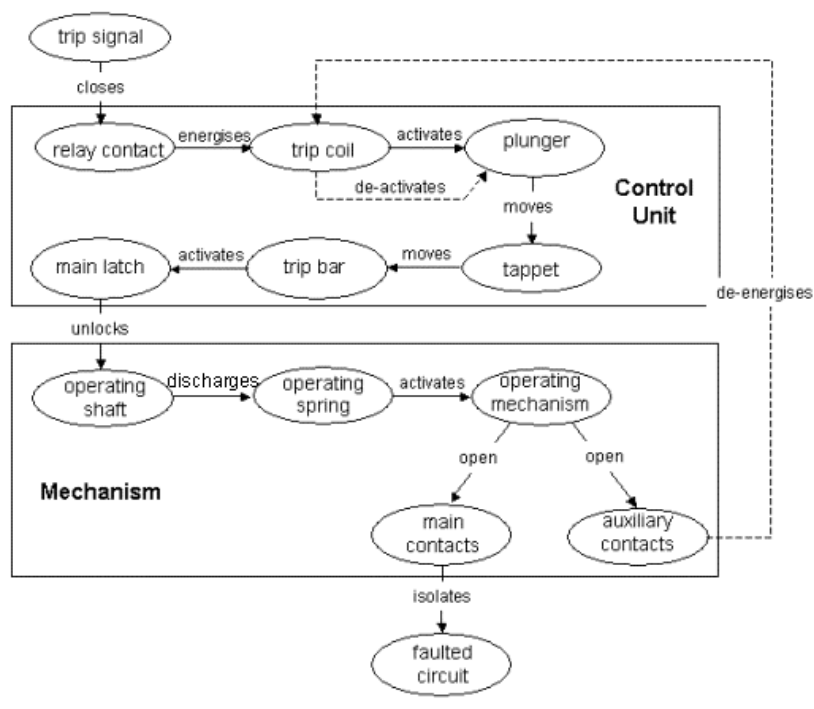

Figure 1 Stages of circuit breaker operation

The current flowing through the circuit breaker trip coil generates a magnetic field affecting a force upon the plunger, moving it towards the breaker latch mechanism. At the same time, the motion of the iron plunger induces an e.m.f. in the coil. Figure 2 illustrates the operation of the control unit's trip coil plunger mechanism and its effect on the trip coil current signature, based on information in [5].

When the trip command is initiated this signal activates a relay, closing a contact connecting the circuit breaker's control circuit, including the trip coil, to the substation d.c. battery supply. As the trip coil is energized by the battery supply and the current rises causing a magnetic field to apply a force on the iron plunger Figure 2 (a). When the force on the plunger exceeds that of stiction the plunger begins to move (Figure 2 (b)) and ' $A$ ' on trip signature). The motion of the iron plunger induces an e.m.f. in the coil effectively reducing the current flowing through it, as indicated by the first current peak (Figure 2 'B') [7].

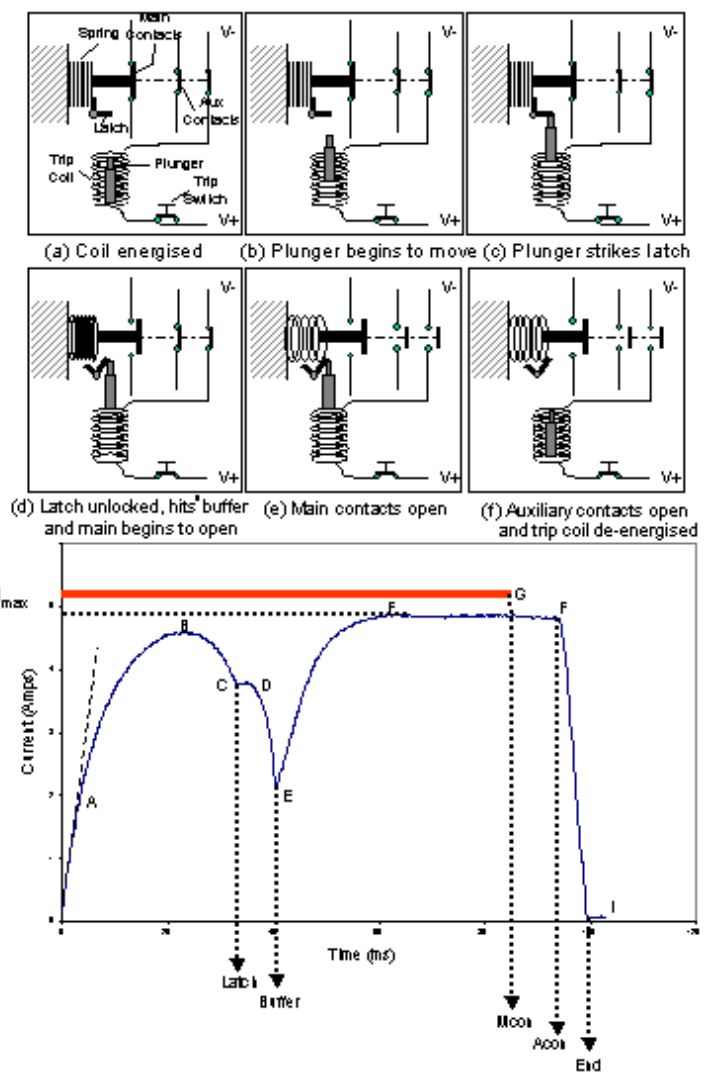

Figure 2 Circuit breaker control circuit operation and captured trip coil current signature, based on [5]

As the plunger continues to accelerate through the coil the current flowing through the trip coil continues to fall (Figure 2 ' $\mathrm{B}$ ' to ' $\mathrm{C}$ '), until the plunger eventually strikes the latch mechanism (Figure 2 (c)), where a sudden reduction in the velocity of the plunger occurs resulting in a 'corner' in the current signature (Figure 2 ' $C$ ' to ' $D$ '). The combined mass of the plunger and the latch reduces the plunger's momentum causing a further reduction in the coil current (Figure 2 ' $\mathrm{D}$ ' to 'E') until it hits a buffer bringing it to rest (Figure 2 (d) 'E'). With the plunger at rest the current increases to the maximum rating of the coil (Figure 2 ' $F$ '). Meanwhile, the latch unlocks the spring operating mechanism, releasing the stored energy required to open the main contacts (Figure 2 (e) 'G'). The trigger for the main contact time captured by the handheld device relies on the current signal taken from a clip-on ammeter clamped around the CT arrangement measuring the current flowing in the conductor on the load side of the breaker. Subsequently when current ceases to flow through this conductor this is recognized by the CT and clip-on ammeter, 
and the corresponding 'trip time' captured by the device. Typically, after a short delay the auxiliary contacts open, disconnecting the trip coil from the substation d.c. battery supply (Figure 2 ' $\mathrm{H}$ '). As the coil is de-energized the trip coil current decays quickly to zero in accordance with the coil inductance (Figure 2 'I'), causing the plunger to return to its initial position (Figure 2 (f)) [7].

Domain knowledge relating to the circuit breaker operation and the idiosyncrasies associated with various manufacturers and models was made available from utility switchgear experts. However, due to the novelty of this condition monitoring technology there remained a fundamental lack of trip signature interpretation expertise.

Through a combination of knowledge elicitation and data mining activity, expert understanding of the breaker operation and behavior could be correlated with observations made from the outcomes of the data analysis conducted.

While the organization's switchgear experts were undeniably knowledgeable in the mechanics of circuit breaker operation and their associated defects and problems, they had no understanding of how this mapped onto associated trip signatures. Due to the novelty and unfamiliarity of this condition monitoring technology, switchgear experts were unable to recognize symptoms or causes of deteriorating breaker condition from the characteristics of a trip signature. Using a structured method of data mining, distinct clusters of trip signature shapes could be identified and quantified for various models and manufacturers encompassing the entire asset base of distribution circuit breakers. These clusters notionally represent different circuit breaker conditions. Further analysis enabled the definition of symptoms characterizing these, as yet, unexplained clusters (i.e. conditions). Using the practical knowledge of experienced switchgear experts to explain the data observations derived from the data mining process, condition assessment and ultimately diagnostic rules could be derived and implemented in a rule-based system.

A prototype was developed which presents, in a visually appreciable format to the data analyst and maintenance expert, the outcomes of the data analysis conducted on a population of captured circuit breaker trip signatures. This allows the expert to define the operational thresholds characterizing the varying degrees of breaker condition observed in the data. This has also led to tentative steps being taken to elicit basic diagnostic knowledge, and so extending the functionality of the decision support system from one of circuit breaker condition assessment to one offering more detailed defect diagnosis and location.

\section{A STRUCTURED METHOD OF MINING CIRCUIT BREAKER TRIP SIGNATURE DATA}

Data mining is often cited as "the non-trivial extraction of implicit, previously unknown, and potentially useful information from data." [8]. Data mining may therefore be considered a knowledge extraction process aimed at "making sense" of large data sets through the discovery of hidden and often unsuspected facts, patterns and correlations implicit in the data. This is achieved by analyzing large volumes of data through a combination of advanced processing techniques, including machine learning, statistical methods and visualization tools. Using these techniques, the observations gleaned from the data provide an insight into the behavior of the system responsible for bearing the data. Knowledge and understanding of the system behavior may in turn be elicited from domain experts and used to infer rules or define models enabling the prediction or classification of future system behavior and/or condition. The method of deploying the 'discovered knowledge' output from the data mining and knowledge elicitation processes may vary from something as simple as circulating a paper-based report detailing the findings of the analysis to a more proactive approach where the knowledge may be embedded within an intelligent decision support system.

This paper proposes a structured method of data mining, customized to meet the data mining goals of plant condition monitoring specifically. The discovered knowledge relating to plant condition assessment and defect diagnosis can then be used to develop a decision support tool offering maintenance staff advice in the condition assessment of distribution circuit breakers following routine trip testing.

In the condition monitoring domain a priori knowledge of the plant condition associated with acquired condition monitoring data may or may not be readily available (e.g. data representing healthy/unhealthy conditions may be labeled or unlabelled). When data-class associations are known, classifier models may be built using supervised learning techniques and incorporated directly into a decision support system (e.g. using labeled data to train an artificial neural network to identify normal and abnormal plant operation). Where data-class associations are unknown, a more complex data mining process is required to extract hidden associations between the data characteristics and those of the plant behavior (i.e. the class). Figure 3 illustrates the data mining objectives (defined by CRISP-DM [9]) involved in developing a classifier model where the data-class associations are unknown prior to developing a classifier model.

The objectives at each stage of this data mining process are described as follows:

- Segmentation - separation of captured condition monitoring data into interesting subgroups representing concepts (e.g. clusters representing varying plant condition/behavior).

- Concept Description - describing the thresholds defining different plant condition and behavior (i.e. quantitative description of the identified clusters).

- Expert interpretation - using domain knowledge, where it exists, to verify and explain the observations made and concepts described from the segmented data.

- Classification - building classifier models or deriving rules to correctly assign conditions to previously 
unseen plant data.

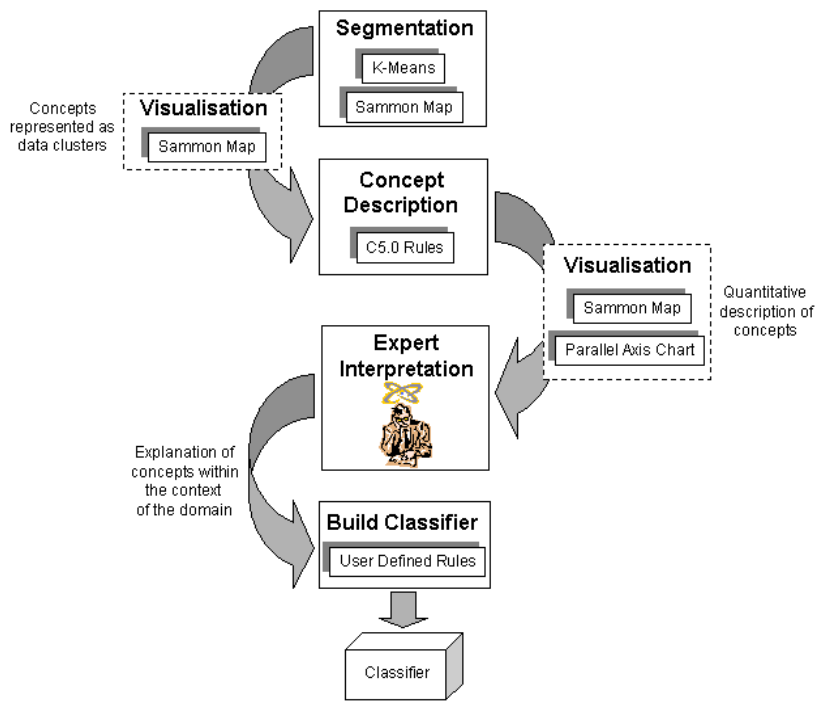

Figure 3 Data mining process for trip signature data analysis and classifier development 